Research paper

\title{
Surface effect in the bending of nanowires
}

\author{
Yin Yao ${ }^{a}$, Shaohua Chen ${ }^{\mathrm{b}, *}$ \\ a LNM, Institute of Mechanics, Chinese Academy of Sciences, Beijing 100190, China \\ ${ }^{\mathrm{b}}$ Institute of Advanced Structure Technology, Beijing Institute of Technology, Beijing 100081, China
}

\section{A R T I C L E I N F O}

\section{Article history:}

Received 8 October 2015

Revised 27 May 2016

Available online 7 June 2016

\section{Keywords:}

Surface effect

Nanowire

Bending

Surface energy density

Effective elastic modulus

\begin{abstract}
A B S T R A C T
The surface effect in the bending of nanowires (nanobeams), including cantilever nanowires and fixedfixed ones, is investigated in this paper with a recently developed elastic theory for nanomaterials, in which only the bulk surface-energy density and the surface-relaxation parameter are involved as two independent parameters to characterize the surface effect. Closed-form solutions of the maximum deflection and the effective elastic modulus in both kinds of nanowires are achieved. It is found that, comparing to the prediction of the classically elastic beam theory, the cantilever nanowire is softened, while the fixed-fixed one is stiffened by the surface effect in nanoscales, consistent well with the existing experimental measurements. Furthermore, an increasing aspect ratio of nanowires can further enhance the stiffening behavior of fixed-fixed nanowires and the softening behavior of cantilever ones, respectively. The present result should be helpful not only for explaining different surface effects in nanowires with different boundary conditions, but also for the design of nano-structures and nano-devices related to nanowires.
\end{abstract}

(c) 2016 Elsevier Ltd. All rights reserved.

\section{Introduction}

The mechanical property of nanowires has attracted considerable interests due to their potential applications in nanostructures and nano-devices, such as sensors and resonators in nano-electromechanical systems (Craighead, 2000; Xie et al., 2012) and reinforcing phases in advanced nanocomposites (Lee et al., 2011; Gong et al., 2013). Similar to the other nanomaterials, nanowires have a size-dependently mechanical behavior due to a large surface-tovolume ratio (Liang and Upmanyu, 2005).

Static bending experiment has been widely adopted to explore the surface effect (size effect) in nanowires' elastic properties. The effective elastic modulus of fixed-fixed nanowires is found to increase with a decreasing diameter of nanowires (Cuenot et al., 2004; Chen et al., 2006; Jing et al., 2006; Tan et al., 2007; Chan et al., 2010; Celik et al., 2011). While for cantilever nanowires, the effective elastic modulus has an oppositely size-dependent behavior (Nam et al., 2006; Gavan et al., 2009; Sadeghian et al., 2009, 2010). All these experimental results provide us a direct understanding of the surface effect (size effect) in nanoscales.

Similar to the size effect in micro-scaled beam bending, which can not be predicted by the classical continuum mechanics, but depends on the strain gradient (for examples, Fleck and Hutchinson (1993, 1997); Gao et al., (1999); Chen and Wang (2000);

\footnotetext{
* Corresponding author.

E-mail address: chenshaohua72@hotmail.com (S. Chen).
}

Gao and Huang, (2001); Chen and Wang (2002), the classical beam theory is also invalid to predict the bending behavior of nanowires. Therefore, an elastic theory considering the surface effect (also addressed as size effect in nanoscales) in nanomaterials should be developed. Fortunately, based on the framework of the surface elasticity theory (Gurtin and Murdoch, 1975, 1978), many investigations on the size-dependently elastic behavior of nanowires have been carried out. Steigmann and Ogden (1997) and Chhapadia et al. (2011) introduced a surface flexural stiffness into the Gurtin-Murdoch (G-M) model in order to describe the curvature-dependent surface energy of bending nanowires. A similar method was also adopted by Chiu and Chen (2011). He and Lilley (2008) applied a generalized Young-Laplace (Y-L) model proposed by Wang and Feng (2007) to study the static bending behavior of nanowires, in which the effect of surface stress induced by a curvature was taken into account. Wang et al. (2010) modeled a bending nanowire as a core-shell composite system, which consists of a surface elastic layer and a core part. Song et al. (2011) improved the Y-L model by considering the in-plane surface stress tangential to the side surface of nanowires. Li et al. (2014) extended the Y-L model to the Timochenko nanobeam case. In addition, the molecular dynamics simulation method, as a major numerical approach, was also adopted to study the bending behavior of nanowires (Park et al., 2005; Chhapadia et al., 2011; Mohammadi and Sharma, 2012; Georgakaki et al., 2014).

The surface elasticity theory as well as its extensions has become a unique and popular model to investigate the surface effect 


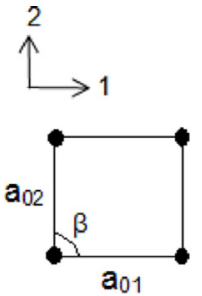

initial configuration

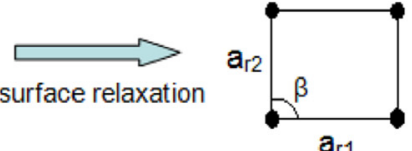

relaxed configuration

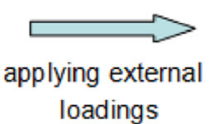

loadings

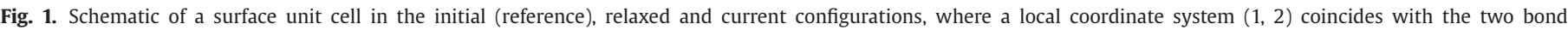
directions.

in the mechanical behavior of nanowires. However, almost all the researchers can not avoid looking for the surface elastic constants involved in the surface elasticity theory, in order to give a precise comparison with the experimental result. Such a process is challenging because no experiment is valid to measure the surface elastic constant till now. Only a few molecular simulations can provide some numerical data (Miller and Shenoy, 2000; Shenoy, 2005; Mi et al., 2008; Chhapadia et al., 2011). Some physical problems of how to achieve the surface elastic constant in molecular simulations still exist and many factors will show significant influences on the numerical data. For example, how many atomic layers in a numerical model should be chosen as the surface of nanomaterials? Is the calculated surface elastic constant affected by the size of the numerical model or the potential energy function? The computational model is atomically continuous in molecular simulations, but an interruption exists between the surface layer and the inside part in most of the theoretical models. Furthermore, a negative value of the surface elastic constant is often found in the molecular simulations (Shenoy, 2005; Mi et al., 2008).

In view of the above problems, a new theory for nanomaterials has been developed recently within the framework of continuum mechanics (Chen and Yao, 2014), in which the surface elastic constant is no longer involved. Instead, a surface-induced traction to characterize the surface effect in nanomaterials is derived, which depends only on the Eulerian surface-energy density. Considering the relationship between the Eulerian surface-energy density and the Lagrangian one yields that only two kinds of material constants are needed in the new elastic theory, i.e., the bulk surfaceenergy density and the surface-relaxation parameter. The former is the surface energy density of a bulk solid while the latter is the ratio of the surface lattice length after and before a spontaneous surface relaxation (Ouyang et al., 2006; Chen and Yao, 2014).

In the present paper, the new theory is further used to analyze the surface effect in the bending of nanowires. Both a cantilever nanowire and a fixed-fixed one are investigated, in which closed-form solutions of the bending deflection and the effective elastic modulus of nanowires are given. Comparison of the theoretical prediction and the experimental result is carried out. The stiffening and softening mechanisms of nanowires with different boundary conditions are further discussed.

\section{Brief introduction of the elastic theory for nanomaterials}

An elastic theory to characterize the surface effect in nanomaterials was proposed by Chen and Yao (2014) recently, which was based on the surface energy density of nanomaterials. Assuming that a nano-solid has an idealized crystal structure, the initial state is regarded as a reference configuration, which will transform into a current one under an external loading. A Lagrangian coordinate system is attached to atoms on the surface with principal axes 1 and 2 parallel to the two basic vectors of a surface unit cell as shown in Fig. 1 (Nix and Gao, 1998). $a_{01}$ and $a_{02}$ represent lattice lengths in the two principal directions, respectively. $\beta$ denotes an angle between the two basic vectors. Due to a spontaneous surface relaxation, two lattice lengths become $a_{r 1}$ and $a_{r 2}$, respectively. Both of them further change to be $a_{1}$ and $a_{2}$ in the current configuration when an external loading is added on the nano-solid.

The potential energy function $\Pi$ of the nano-solid in the current configuration can be written as

$\Pi(\mathbf{u})=\int_{V-S} \psi(\varepsilon) d V+\int_{S} \phi d S-\int_{V-S} \mathbf{f} \cdot \mathbf{u} d V-\int_{S_{p}} \mathbf{p} \cdot \mathbf{u} d S$

where $\psi$ is the elastic strain energy density, $\phi$ is the Eulerian surface-energy density in the current configuration, $\mathbf{f}$ and $\mathbf{p}$ are the body force and external surface traction, respectively. $\mathbf{u}$ and $\varepsilon$ are the displacement and strain induced by $\mathbf{f}$ and $\mathbf{p}$. $V$ and $S$ denote the volume and the surface of the nano-solid.

The variation analysis of Eq. (1) yields the following equilibrium equation and stress boundary conditions,

$\left\{\begin{array}{l}\boldsymbol{\sigma} \cdot \nabla+\mathbf{f}=0(\text { in } V-S) \\ \mathbf{n} \cdot \boldsymbol{\sigma} \cdot \mathbf{n}=\mathbf{p} \cdot \mathbf{n}-\gamma_{n} \mathbf{n}(\text { on } S) \\ (\mathbf{I}-\mathbf{n} \otimes \mathbf{n}) \cdot \boldsymbol{\sigma} \cdot \mathbf{n}=(\mathbf{I}-\mathbf{n} \otimes \mathbf{n}) \cdot \mathbf{p}-\gamma_{t}(\text { on } S)\end{array}\right.$

where $\sigma$ is the bulk Cauchy stress tensor, $\nabla$ is a spatial gradient operator in the current configuration, $\mathbf{n}$ is the unit normal vector perpendicular to the surface $S$ of the nano-solid, $\mathbf{I}$ is a unit tensor; $\gamma_{n}$ and $\gamma_{t}$ are the normal and tangential components of an additionally surface-induced traction vector, respectively, which characterizes a force disturbance at boundaries due to the surface effect. Based on an infinitesimal element, the virtual work method yields the surface-induced traction as (Chen and Yao, 2014),

$\gamma_{t}=\nabla_{s} \phi, \gamma_{n} \mathbf{n}=\phi\left(\frac{1}{R_{1}}+\frac{1}{R_{2}}\right) \mathbf{n}=\phi\left(\mathbf{n} \cdot \nabla_{s}\right) \mathbf{n}$

where $\nabla_{S}$ is a surface gradient operator in the current configuration, $R_{1}$ and $R_{2}$ are the two principal radii of curvature of a curved surface.

Relation between the Eulerian surface-energy density $\phi$ and the Lagrangian surface energy density $\phi_{0}$ satisfies

$\phi=\frac{\phi_{0}}{J_{s}}$

where $J_{s}$ is a Jacobean determinant characterizing the surface deformation from the reference configuration to the current one. Eq. (4) can also be found in Nix and Gao (1998) and Huang and Wang (2006).

Thus, the equilibrium equations can be rewritten as (Chen and Yao, 2014),

$$
\left\{\begin{array}{l}
\boldsymbol{\sigma} \cdot \nabla+\mathbf{f}=0(\text { in } V-S) \\
\mathbf{n} \cdot \boldsymbol{\sigma} \cdot \mathbf{n}=\mathbf{p} \cdot \mathbf{n}-\frac{\phi_{0}\left(\mathbf{n} \cdot \nabla_{s}\right)}{J_{s}}(\text { on } S) \\
(\mathbf{I}-\mathbf{n} \otimes \mathbf{n}) \cdot \boldsymbol{\sigma} \cdot \mathbf{n}=(\mathbf{I}-\mathbf{n} \otimes \mathbf{n}) \cdot \mathbf{p}+\frac{\phi_{0}\left(\nabla_{S} J_{s}\right)}{J_{S}^{2}}-\frac{\nabla_{s} \phi_{0}}{J_{S}}(\text { on } S)
\end{array}\right.
$$




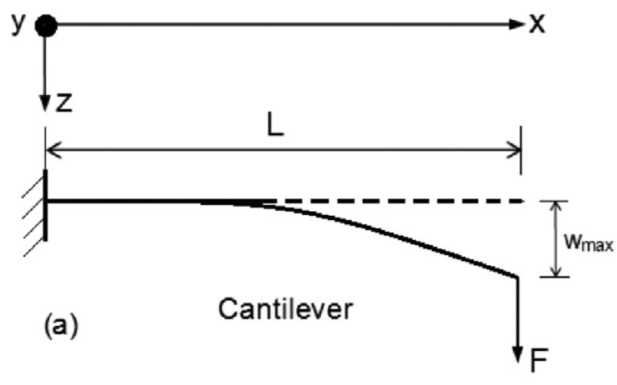

(c)

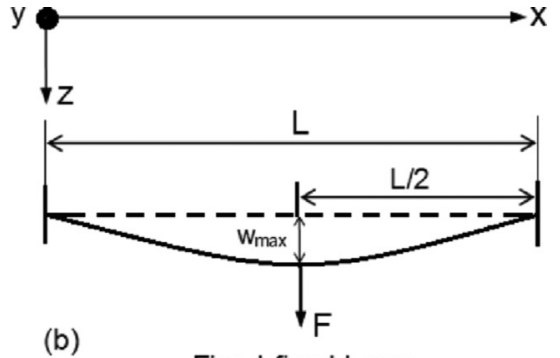

Fixed-fixed beam
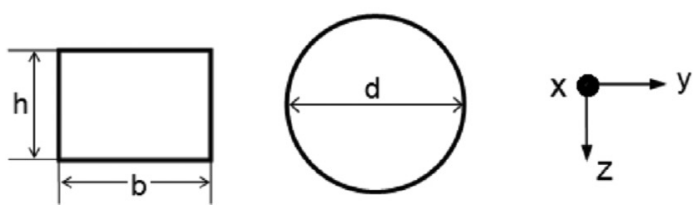

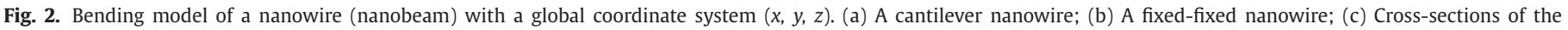
two kinds of nanowires.

The Lagrangian surface energy density $\phi_{0}$ in the reference configuration consists of a structural part $\phi_{0}^{\text {stru }}$ related to the surface strain energy and a chemical part $\phi_{0}^{\text {chem }}$ originating from the surface dangling-bond energy (Ouyang et al., 2006, 2008; Mi et al., 2008),

$$
\begin{aligned}
\phi_{0}= & \phi_{0}^{\text {stru }}+\phi_{0}^{\text {chem }} \\
\phi_{0}^{\text {stru }}= & \frac{E_{b}}{2 \sin \beta} \sum_{i=1}^{2} a_{0 i} \eta_{i}\left\{\left[3+\left(\lambda_{i}+\lambda_{i} \varepsilon_{s i}\right)^{-m}-3\left(\lambda_{i}+\lambda_{i} \varepsilon_{s i}\right)\right]\right. \\
& \left.\times\left[\lambda_{i}^{2} \varepsilon_{s i}^{2}+\left(\lambda_{i}-1\right)^{2}+2 \lambda_{i}\left(\lambda_{i}-1\right) \varepsilon_{s i}\right]\right\} \\
\phi_{0}^{\text {chem }}= & \phi_{0 b}\left(1-w_{1} \frac{D_{0}}{D}\right), \eta_{1}=a_{01} / a_{02}, \eta_{2}=a_{02} / a_{01}
\end{aligned}
$$

where $\phi_{0 b}$ is the surface energy density of bulk materials, $D_{0}$ is a critical size $\left(D_{0}=3 d_{a}\right.$ for nanoparticles and $2 d_{a}$ for nano-thin films, where $d_{a}$ is the atomic diameter), $D$ is a characteristic scale of nanomaterials (e.g., thickness, diameter, etc.). $w_{1}$ is a parameter governing the size-dependent behavior of $\phi_{0}^{\text {chem }}$ (Chen and Yao, 2014). $E_{b}$ is the Young's modulus of bulk materials, $\lambda_{i}=a_{r i} / a_{0 i}$ denotes the surface relaxation parameter, $\varepsilon_{s i}=\left(a_{i}-a_{r i}\right) / a_{r i}$ is the surface strain induced only by the external loading. $m$ is a parameter describing the dependence of bond lengths on the binding energy ( $m=4$ for alloys or compounds and $m=1$ for pure metals) (Sun, 2003).

In contrast to the G-M theory (Gurtin and Murdoch, 1975), the new theory no longer requires the surface elastic constant. The Lagrangian surface-energy density $\phi_{0}$ in the reference configuration serves as a unique quantity characterizing the surface effect of nanomaterials, which depends on the bulk surface-energy density and the relaxation parameter. Both parameters have clearly physical meanings and can be determined very easily through experiment and simple MD simulation.

\section{Surface effect in the bending of nanowires}

Fig. 2 shows a nanowire of length $L$ with the longitudinal axis in the $x$ direction and the bending deflection in the $z$ direction. The cross section of the nanowire can be rectangular with a height $h$ and a width $b(b \geq h)$, or circular with a diameter $d$. Both a cantilever nanowire and a fixed-fixed one are analyzed in this paper by the elastic theory for nanomaterials.

\subsection{The potential energy function of a bending nanowire}

According to Timoshenko and Gere (1972), the relation among the axial displacement $u_{x}$, the bending deflection $w$ and the axial strain $\varepsilon_{X}$ for an Euler-Bernoulli beam, can be expressed as

$u_{x}=-z \frac{d w}{d x}, \varepsilon_{x}=-z \frac{d^{2} w}{d x^{2}}, 0 \leq x \leq L$

The variation of the bulk strain energy $U$ can be written as

$$
\delta U=\delta \int_{V} \frac{1}{2} \sigma_{x} \varepsilon_{x} d V=\delta \int_{0}^{L} \frac{1}{2} E_{b} I\left(\frac{d^{2} w}{d x^{2}}\right)^{2} d x=\int_{0}^{L} E_{b} I \frac{d^{2} w}{d x^{2}} \frac{d^{2}(\delta w)}{d x^{2}} d x
$$

where $I=\int_{A_{n w}} z^{2} d A$ is the inertia moment and $A_{n w}$ denotes the area of nanowires' cross-section.

The variation of the surface energy can be written as (Zhang et al., 2010)

$\delta \Phi=\int_{S_{n w}} \gamma \cdot \delta \mathbf{u} d S=\int_{0}^{L} d x \int_{C_{n w}}\left(\gamma_{t} \cdot \delta \mathbf{u}_{t}+\gamma_{n} \delta u_{n}\right) d C$

where $S_{n w}$ represents the lateral surface of the nanowire, $C_{n w}$ is the perimeter of the nanowire's cross section. $\delta \mathbf{u}_{t}$ and $\delta u_{n}$ are the tangential and normal displacement components of $\delta \mathbf{u}$, respectively. The vector $\boldsymbol{\gamma}_{t}$ can be decomposed into a component $\gamma_{x}$ in the axial direction and a component $\gamma_{c}$ in the circumferential direction on the surface. For a thin nanowire, the latter is often neglected (Chen and Chiu, 2011; Song et al., 2011). Combining Eqs. (3), (4) and (7) leads to

$\gamma_{t} \cdot \delta \mathbf{u}_{t}=\gamma_{x} \delta u_{x}=\frac{\partial \phi}{\partial x}\left[-z \frac{d(\delta w)}{d x}\right]=\left(\frac{1}{J_{s}} \frac{\partial \phi_{0}}{\partial x}-\frac{\phi_{0}}{J_{s}^{2}} \frac{\partial J_{s}}{\partial x}\right)\left[-z \frac{d(\delta w)}{d x}\right]$

A real nanowire may have different crystal facet orientations on its lateral surfaces from its axially oriented surface. For example, $\mathrm{a}<100>$ axially oriented nanowire may have $\{111\}$ and $\{112\}$ lateral surface orientations. In this case, the surface energy density of different crystal surfaces leads to different surface-induced tractions as given by Eq. (3). On some crystal surfaces, the atomic spacing and relaxation in two bond directions may not be the same (e.g. $\{110\}$ surface) also. For such a nanowire with anisotropic surfaces, the total surface energy should be characterized by the sum of surface energies of different oriented facets. For simplicity, a [100] axially-oriented nanowire with the same crystal facet lateral surfaces is considered, which has an equal atom spacing in 
both bond directions (He and Lilley, 2008; Chhapadia et al, 2011; Song et al, 2011) and is assumed as a perfectly and isotropically cylindrical surface in the theoretical analysis, as done in the surface elasticity models (Wang and Feng, 2007; He and Lilley, 2008; Song et al., 2011; Wolfer, 2011). As a result, we have $\beta=90^{\circ}$ and $a_{01}=a_{02}=\sqrt{2} a_{0} / 2$, where $a_{0}$ is the lattice constant of a bulk material. The surface relaxation in both bond directions is the same, i.e., $\lambda_{1}=\lambda_{2}=\lambda$ (Ouyang et al, 2006). Meanwhile, the surface strain $\varepsilon_{s i}(i=1,2)$ in both bond directions equals $\varepsilon_{x} / 2$ (Details are given in Appendix). For a metallic nanowire, we have $m=1$, $D=d$ or $h, D_{0}=3 d_{a}$ and $w_{1}=1 / 4$ (Jiang et al., 2001; Sun, 2003; Liang et al., 2010). Then, Eq. (6) is rewritten as

$$
\begin{aligned}
\phi_{0}= & \phi_{0 b}\left(1-\frac{3 d_{a}}{4 D}\right)+\frac{\sqrt{2} E_{b} a_{0}}{2}\left[3+\frac{1}{\lambda\left(1+\varepsilon_{x} / 2\right)}-3\left(\lambda+\frac{\lambda \varepsilon_{x}}{2}\right)\right] \\
& \times\left[\frac{\lambda^{2} \varepsilon_{x}^{2}}{4}+2 \lambda(\lambda-1) \frac{\varepsilon_{x}}{2}+(\lambda-1)^{2}\right]
\end{aligned}
$$

The Jacobean determinant related to the surface deformation is $J_{s}=\lambda^{2}\left(1+\varepsilon_{x} / 2\right)^{2}$. Combining Eqs. (3), (7), (10), (11) and using Taylor series approximation yield the surface-induced traction $\gamma_{x}$,

$\gamma_{x}=\left[C_{0} z+C_{1} z^{2} \frac{d^{2} w}{d x^{2}}+C_{2} z^{3}\left(\frac{d^{2} w}{d x^{2}}\right)^{2}\right] \frac{d^{3} w}{d x^{3}}$

where

$$
\begin{aligned}
C_{0}= & \phi_{0}^{*}(5-4 \lambda)-\frac{\sqrt{2} E_{b} a_{0} A_{2}(3-2 \lambda)}{2} \\
C_{1}= & 2 \phi_{0}^{*}+\sqrt{2} E_{b} a_{0} A_{1}(3-2 \lambda)-\frac{\sqrt{2} E_{b} a_{0} A_{2}(5-4 \lambda)}{2} \\
C_{2}= & \frac{\sqrt{2} E_{b} a_{0} A_{1}(7-4 \lambda)}{2}-\sqrt{2} E_{b} a_{0} A_{2}, \phi_{0}^{*}=\phi_{0 b}\left(1-\frac{3 d_{a}}{4 D}\right) \\
& +\frac{\sqrt{2} E_{b} a_{0}}{2}(\lambda-1)^{2} \\
A_{1}= & \frac{1-10(\lambda-1)-17(\lambda-1)^{2}}{4}, A_{2}=(\lambda-1)-5(\lambda-1)^{2}
\end{aligned}
$$

Considering Eqs. (3), (4), (11) and using the curvature $\kappa=-(\mathbf{n}$. $\left.\nabla_{s}\right)=d^{2} w / d x^{2}, \delta u_{n} \approx \delta w$ (Chen and Chiu, 2011; Song et al., 2011), we have

$$
\begin{aligned}
\gamma_{n} \delta u_{n} & \approx-\phi \kappa \delta w=-\frac{\phi_{0}}{J_{s}} \frac{d^{2} w}{d x^{2}} \delta w \\
& =-\left[D_{0} z+D_{1} z \frac{d^{2} w}{d x^{2}}+D_{2} z^{2}\left(\frac{d^{2} w}{d x^{2}}\right)^{2}+D_{3} z^{3}\left(\frac{d^{2} w}{d x^{2}}\right)^{3}\right] \frac{d^{2} w}{d x^{2}} \delta w
\end{aligned}
$$

where

$$
\begin{aligned}
& D_{0}=\phi_{0}^{*}(3-2 \lambda), D_{1}=\phi_{0}^{*}-\frac{\sqrt{2} E_{b} a_{0} A_{2}(3-2 \lambda)}{2} \\
& D_{2}=\frac{\sqrt{2} E_{b} a_{0} A_{1}(3-2 \lambda)}{2}-\sqrt{2} E_{b} a_{0} A_{2}, D_{3}=\frac{\sqrt{2} E_{b} a_{0} A_{1}}{2}
\end{aligned}
$$

Then, the variation of the potential energy function can be written as,

$$
\begin{aligned}
\delta \Pi= & \delta U+\delta \Phi-\delta W \\
= & \int_{0}^{L} E_{b} I\left(\frac{d^{2} w}{d x^{2}}\right) \frac{d^{2}(\delta w)}{d x^{2}} d x \\
& +\int_{0}^{L} d x \int_{C_{n w}}\left(\gamma_{x} \delta u_{x}+\gamma_{n} \delta u_{n}\right) d C-F \delta w_{\max } \\
= & \int_{0}^{L} E_{b} I\left(\frac{d^{2} w}{d x^{2}}\right) \frac{d^{2}(\delta w)}{d x^{2}} d x
\end{aligned}
$$

$$
\begin{aligned}
& -\int_{0}^{L}\left[C_{0} I_{s 1}+C_{2} I_{s 2}\left(\frac{d^{2} w}{d x^{2}}\right)^{2}\right] \frac{d^{3} w}{d x^{3}} \frac{d(\delta w)}{d x} d x \\
& -\int_{0}^{L}\left[D_{0} I_{c}+D_{2} I_{s 1}\left(\frac{d^{2} w}{d x^{2}}\right)^{2}\right] \frac{d^{2} w}{d x^{2}} \delta w d x-F \delta w_{\text {max }}
\end{aligned}
$$

in which $I_{s 1}=\int_{C_{N W}} z^{2} d C, I_{s 2}=\int_{C_{N W}} z^{4} d C$ and $I_{C}=\int_{C_{n w}} n_{w}^{2} d C$. Here $n_{w}$ represents the vertical component of the unit normal vector $\mathbf{n}$, which is parallel to $w(x)$.

For nanowires with different cross-section shapes, we have

Rectangular $: I=\frac{b h^{3}}{12}, I_{s 1}=\frac{b h^{2}}{2}+\frac{h^{3}}{6}, I_{s 2}=\frac{b h^{4}}{8}+\frac{h^{5}}{80}, I_{c}=2 b$

Circular $: I=\frac{\pi d^{4}}{64}, I_{s 1}=\frac{\pi d^{3}}{8}, I_{s 1}=\frac{3 \pi d^{5}}{128}, I_{c}=\frac{\pi d}{2}$

\subsection{Closed-form solution of a fixed-fixed nanowire}

For a fixed-fixed nanowire, both the end at $x=0$ and that at $x=L$ are clamped with a concentrated force $F$ acting at $x=L / 2$. The maximum bending deflection is denoted as $w_{\max }$, which occurs at $x=L / 2$. Due to the symmetric characteristic of the bending model, only half of the nanowire is considered. Ignoring the highorder terms results in the variation of the potential energy,

$$
\begin{aligned}
\delta \Pi= & \int_{0}^{L / 2}\left[\left(E_{b} I+C_{0} I_{s 1}\right) \frac{d^{4} w}{d x^{4}}-D_{0} I_{c} \frac{d^{2} w}{d x^{2}}\right] \delta w d x \\
& +\left[E_{b} I \frac{d^{2} w}{d x^{2}} \frac{d(\delta w)}{d x}\right]_{x=0}^{x=L / 2} \\
& -\left[\left(E_{b} I+C_{0} I_{s 1}\right) \frac{d^{3} w}{d x^{3}} \delta w\right]_{x=0}^{x=L / 2}-\frac{F}{2}(\delta w)_{x=L / 2}
\end{aligned}
$$

Let $\delta \Pi=0$. Then, considering the boundary conditions $w(0)=$ $w(L)=0$ and $w^{\prime}(0)=w^{\prime}(L / 2)=w^{\prime}(L)=0$ yields

$$
\begin{aligned}
& \left(E_{b} I+C_{0} I_{s 1}\right) \frac{d^{4} w}{d x^{4}}-D_{0} I_{c} \frac{d^{2} w}{d x^{2}}=0 \\
& {\left[\left(E_{b} I+C_{0} I_{s 1}\right) \frac{d^{3} w}{d x^{3}}\right]_{x=L / 2}=-\frac{F}{2}}
\end{aligned}
$$

which are the deflection equation and the force boundary condition for the bending of a fixed-fixed nanowire, respectively.

For a wire (beam) with a relatively large characteristic scale, the surface effect is negligible, i.e., $C_{0}=0$ and $D_{0}=0$. The deflection equation can be well degraded to the classical one, $E_{b} I w^{(4)}(x)=0$ (Timoshenko and Gere, 1972).

The deflection function can be easily solved from Eq. (19),

$w(x)=S_{1}+S_{2} x+S_{3} \cosh (k x)+S_{4} \sinh (k x)$

$$
k=\sqrt{\frac{D_{0} I_{c}}{E_{b} I+C_{0} I_{s 1}}}, 0 \leq x \leq L / 2
$$

Substituting the following boundary conditions into Eq. (20)

$$
\begin{aligned}
& x=0: w=0, \frac{d w}{d x}=0 \\
& x=\frac{L}{2}: \frac{d w}{d x}=0,\left[\left(E_{b} I+C_{0} I_{s 1}\right) \frac{d^{3} w}{d x^{3}}\right]=-\frac{F}{2}
\end{aligned}
$$

yields

$$
\begin{aligned}
& S_{1}=-\frac{F \sqrt{E_{b} I+C_{0} I_{s 1}} \sinh (k L / 4)}{2\left(D_{0} I_{c}\right)^{3 / 2} \cosh (k L / 4)}, \quad S_{2}=\frac{F}{2 D_{0} I_{c}}, \\
& S_{3}=\frac{F \sqrt{E_{b} I+C_{0} I_{s 1}} \sinh (k L / 4)}{2\left(D_{0} I_{c}\right)^{3 / 2} \cosh (k L / 4)}, \quad S_{4}=-\frac{F \sqrt{E_{b} I+C_{0} I_{s 1}}}{2\left(D_{0} I_{c}\right)^{3 / 2}}
\end{aligned}
$$


A closed-form solution of the bending deflection is achieved

$$
\begin{aligned}
w(x)= & -\frac{F \sqrt{E_{b} I+C_{0} I_{s 1}} \sinh (k L / 4)}{2\left(D_{0} I_{c}\right)^{3 / 2} \cosh (k L / 4)}+\frac{F x}{2 D_{0} I_{c}} \\
- & \frac{F \sqrt{E_{b} I+C_{0} I_{s 1}} \sinh [k(x-L / 4)]}{2\left(D_{0} I_{c}\right)^{3 / 2} \cosh (k L / 4)} \\
& (0 \leq x \leq L / 2)
\end{aligned}
$$

with a maximum deflection $w_{\max }$,

$w_{\max }=w(L / 2)=\frac{F L}{4 D_{0} I_{c}}-\frac{F \sqrt{E_{b} I+C_{0} I_{s 1}} \sinh (k L / 4)}{\left(D_{0} I_{c}\right)^{3 / 2} \cosh (k L / 4)}$

Equaling the maximum deflection $w_{\max }$ in Eq. (24) to the classical solution (Chen et al., 2006; Chiu and Chen, 2011), i.e.,

$\frac{F L}{4 D_{0} I_{c}}-\frac{F \sqrt{E_{b} I+C_{0} I_{s 1}} \sinh (k L / 4)}{\left(D_{0} I_{c}\right)^{3 / 2} \cosh (k L / 4)}=\frac{F L^{3}}{192 E_{e f f} I}$

yields a closed-form solution of the effective elastic modulus $E_{\text {eff }}$

$$
E_{\text {eff }}==\frac{L^{3}}{192 I\left[\frac{L}{4 D_{0} I_{c}}-\frac{\sqrt{E_{b} I+C_{0} I_{s 1}} \sinh (k L / 4)}{\left(D_{0} I_{c}\right)^{3 / 2} \cosh (k L / 4)}\right]}
$$

\subsection{Closed-form solution of a cantilever nanowire}

For the bending of a cantilever nanowire with a fixed end at $x=0$ and a concentrated force $F$ acted at the other end $x=L$, the variation of the potential energy can be written as

$$
\begin{aligned}
\delta \Pi= & \int_{0}^{L}\left[\left(E_{b} I+C_{0} I_{s 1}\right) \frac{d^{4} w}{d x^{4}}-D_{0} I_{c} \frac{d^{2} w}{d x^{2}}\right] \delta w d x \\
& +\left[E_{b} I \frac{d^{2} w}{d x^{2}} \frac{d(\delta w)}{d x}\right]_{x=0}^{x=L} \\
& -\left[\left(E_{b} I+C_{0} I_{s 1}\right) \frac{d^{3} w}{d x^{3}} \delta w\right]_{x=0}^{x=L}-F(\delta w)_{x=L}
\end{aligned}
$$

Let $\delta \Pi=0$. Then, considering the boundary conditions $w(0)=$ $0, w^{\prime}(0)=0$, we have

$$
\begin{aligned}
& \left(E_{b} I+C_{0} I_{s 1}\right) \frac{d^{4} w}{d x^{4}}-D_{0} I_{c} \frac{d^{2} w}{d x^{2}}=0 \\
& E_{b} I\left(\frac{d^{2} w}{d x^{2}}\right)_{x=L}=0,\left[\left(E_{b} I+C_{0} I_{s 1}\right) \frac{d^{3} w}{d x^{3}}\right]_{x=L}=-F
\end{aligned}
$$

The deflection function can be easily found from Eq. (28),

$w(x)=S_{1}+S_{2} x+S_{3} \cosh (k x)+S_{4} \sinh (k x)$

$$
k=\sqrt{\frac{D_{0} I_{c}}{E_{b} I+C_{0} I_{s 1}}}, 0 \leq x \leq L
$$

Using the following boundary conditions to solve Eq. (29),

$$
\begin{aligned}
& x=0: w=0, \frac{d w}{d x}=0 \\
& x=L: E_{b} I\left(\frac{d^{2} w}{d x^{2}}\right)=0, \quad\left[\left(E_{b} I+C_{0} I_{s 1}\right) \frac{d^{3} w}{d x^{3}}\right]=-F
\end{aligned}
$$

we have

$$
\begin{aligned}
& S_{1}=-\frac{F \sinh (k L) \sqrt{E_{b} I+C_{0} I_{s 1}}}{\left(D_{0} I_{c}\right)^{3 / 2}}, S_{2}=\frac{F \cosh (k L)}{D_{0} I_{c}}, \\
& S_{3}=\frac{F \sinh (k L) \sqrt{E_{b} I+C_{0} I_{s 1}}}{\left(D_{0} I_{c}\right)^{3 / 2}}, S_{4}=-\frac{F \cosh (k L) \sqrt{E_{b} I+C_{0} I_{s 1}}}{\left(D_{0} I_{c}\right)^{3 / 2}}
\end{aligned}
$$

Table 1

Material parameters involved in our model.

\begin{tabular}{llllll}
\hline & $\mathrm{d}_{\mathrm{a}}(\mathrm{nm})$ & $\mathrm{a}_{0}(\mathrm{~nm})$ & $\mathrm{E}_{\mathrm{b}}(\mathrm{GPa})$ & $\phi_{\mathrm{b}(001)}(\mathrm{N} / \mathrm{m})$ & $\mathrm{c}_{1(001)}(\mathrm{nm})$ \\
\hline $\mathrm{Ag}$ & 0.2889 & 0.418 & 78 & 1.2 & 0.016 \\
$\mathrm{Au}$ & 0.2884 & 0.42 & 79 & 1.63 & 0.025 \\
$\mathrm{~Pb}$ & 0.36 & 0.5 & 16 & 0.6 & $/$ \\
$\mathrm{Si}$ & 0.22 & 0.54 & 169 & 2.2 & $/$ \\
\hline
\end{tabular}

A closed-form solution of the bending deflection can be found

$$
\begin{aligned}
w(x)= & -\frac{F \sinh (k L) \sqrt{E_{b} I+C_{0} I_{s 1}}}{\left(D_{0} I_{c}\right)^{3 / 2}}+\frac{F \cosh (k L) x}{D_{0} I_{c}} \\
& +\frac{F \sqrt{E_{b} I+C_{0} I_{s 1}}}{\left(D_{0} I_{c}\right)^{3 / 2}} \sinh [k(L-x)] \\
& (0 \leq x \leq L)
\end{aligned}
$$

with the maximum deflection $w_{\max }$ at $x=L$,

$w_{\max }=w(L)=\frac{F \cosh (k L) L}{D_{0} I_{c}}-\frac{F \sinh (k L) \sqrt{E_{b} I+C_{0} I_{s 1}}}{\left(D_{0} I_{c}\right)^{3 / 2}}$

Similar to the fixed-fixed nanowire case, equaling the maximum deflection in Eq. (33) to the classical solution

$$
\frac{F \cosh (k L) L}{D_{0} I_{c}}-\frac{F \sinh (k L) \sqrt{E_{b} I+C_{0} I_{s 1}}}{\left(D_{0} I_{c}\right)^{3 / 2}}=\frac{F L^{3}}{3 E_{e f f} I}
$$

yields a closed-form solution of the effective elastic modulus $E_{\text {eff }}$ for the bending of a cantilever nanowire,

$$
E_{e f f}==\frac{L^{3}}{3 I\left[\frac{\cosh (k L) L}{D_{0} I_{c}}-\frac{\sinh (k L) \sqrt{E_{b} I+C_{0} I_{s 1}}}{\left(D_{0} I_{c}\right)^{3 / 2}}\right]}
$$

\section{Results and discussion}

The deflection and the effective elastic modulus of silver, gold, lead and silicon nanowires with (001) surfaces will be predicted theoretically using the present model, in which the surface relaxation parameter $\lambda$ is proportional to the height $h$ or diameter $d$ of nanowires, $\lambda=1-c_{r} / D\left(c_{r}>0, D=h o r d\right)$ (Diao et al., 2004; Ouyang et al, 2008; Olsson and Park, 2012). The value of $c_{r}$ and the other material parameters involved in the present model are given in Table 1 (Jaccodine, 1963; Sadeghian et al., 2009; Sheng et al., 2011). $\lambda$ approaches one for nanowires with a relatively large characteristic size.

\subsection{The case of a fixed-fixed nanowire}

The deflection of a fixed-fixed silver ( $\mathrm{Ag}$ ) nanowire is predicted theoretically with our model and compared with the existing experimental result given by Chen et al. (2006) as shown in Fig. 3, in which theoretical results obtained by the Young-Laplace (Y-L) model (He and Lilley, 2008) and the classical beam theory (Timoshenko and Gere, 1972) are also shown for comparison. All the material parameters are taken from the experiment work (Chen et al., 2006), such as $L=1994 \mathrm{~nm}, d=65.9 \mathrm{~nm}, F=62 \mathrm{nN}$. The involved surface elastic modulus $E_{S}$ in the Y-L model is taken as $1.22 \mathrm{~N} / \mathrm{m}$ (He and Lilley, 2008). Fig. 3 shows that the theoretical results considering the surface effect agree well with the experimental one, while the classical one deviates obviously from the experimental measurement, especially for the maximum deflection. The difference of the maximum deflection predicted by the present theoretical model and the experimental one is less than $2 \%$. Both the present theoretical prediction and the experimental 

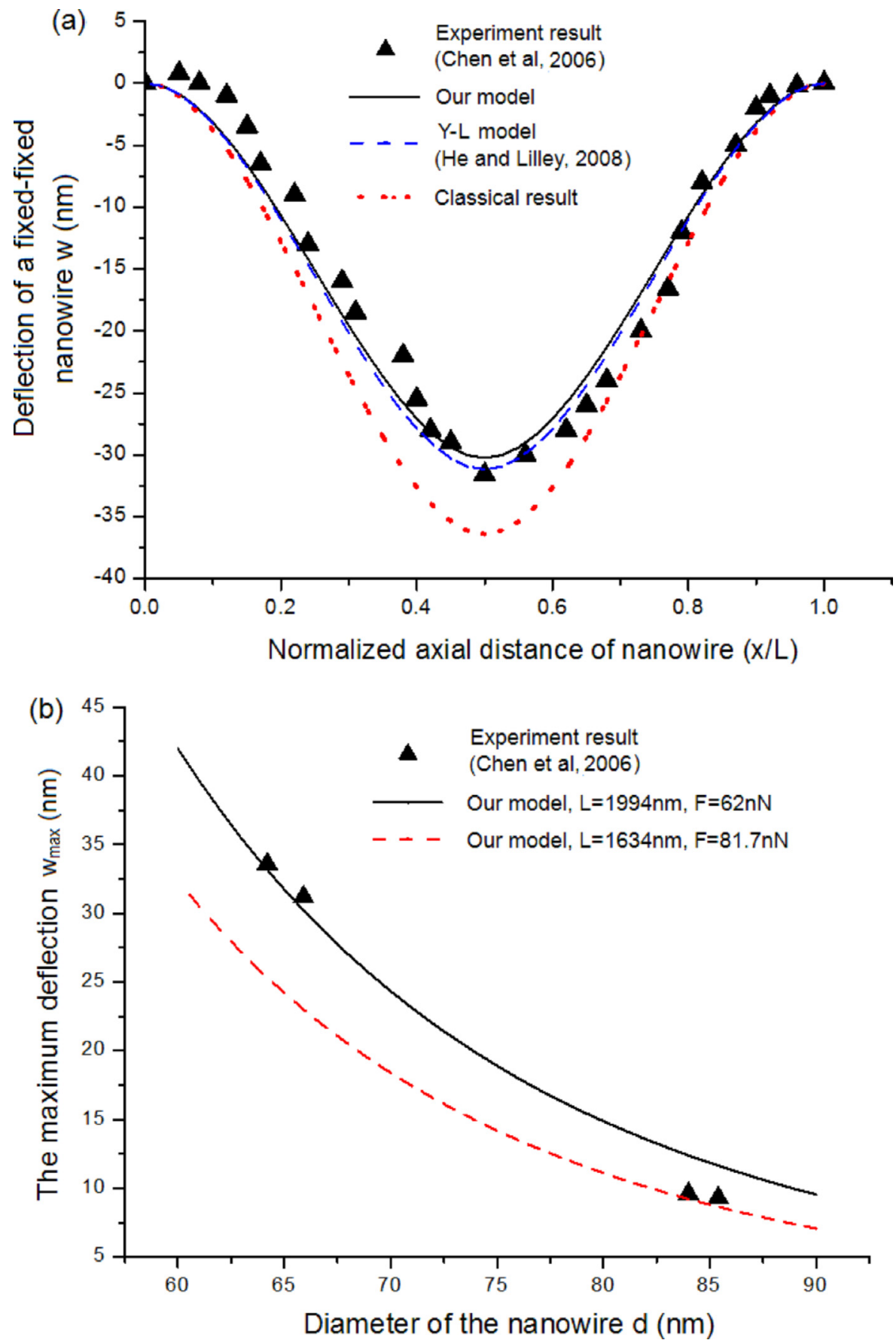

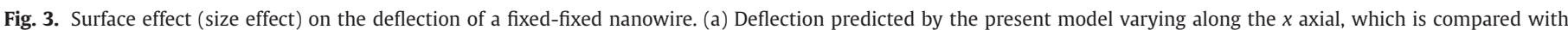

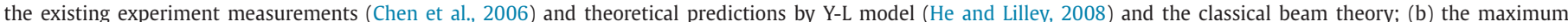
deflection as a function of the diameter of nanowires, where the prediction of the present model is compared with the existing experimental results (Chen et al., 2006).

measurement demonstrate that the bending stiffness of a fixedfixed nanowire is effectively enhanced due to the surface effect at nanoscale. Comparison of the maximum deflection predicted by the theory for nanomaterials and the one measured experimentally is given in Fig. 3(b) for nanowires of different lengths and diameters, which shows a good agreement also.

The effective elastic modulus $E_{\text {eff }}$ is shown in Fig. 4, where the theoretical result is compared with the experimental one given by Cuenot et al. (2004) for a lead (Pb) nanowire and Jing et al. (2006) for a silver (Ag) nanowire, respectively. The theoretical result predicted by the present model agrees very well with the experimental measurement, even for lead nanowires with a diameter less than $70 \mathrm{~nm}$ and silver nanowires with a diameter less than $30 \mathrm{~nm}$, whose effective elastic modulus is approximately twice the corresponding bulk value (Cuenot et al., 2004; Jing et al., 2006). Both the theoretical and experimental results show that the effective elastic modulus $E_{\text {eff }}$ tends to the bulk one and is almost insensitive to the diameter $d$ when the diameter of nanowires is large enough, for example, $d>110 \mathrm{~nm}$ for $\mathrm{Pb}$ and $d>60 \mathrm{nmfor}$ Ag.

The same problem was also investigated by Miller and Shenoy (2000) and Chhapadia et al. (2011), in which the effective elastic modulus was expressed as

$E_{e f f}=E_{b}\left(1+\frac{E_{s} I_{s 1}}{E_{b} I}\right),(\mathrm{M}-$ Smodel $)$ 

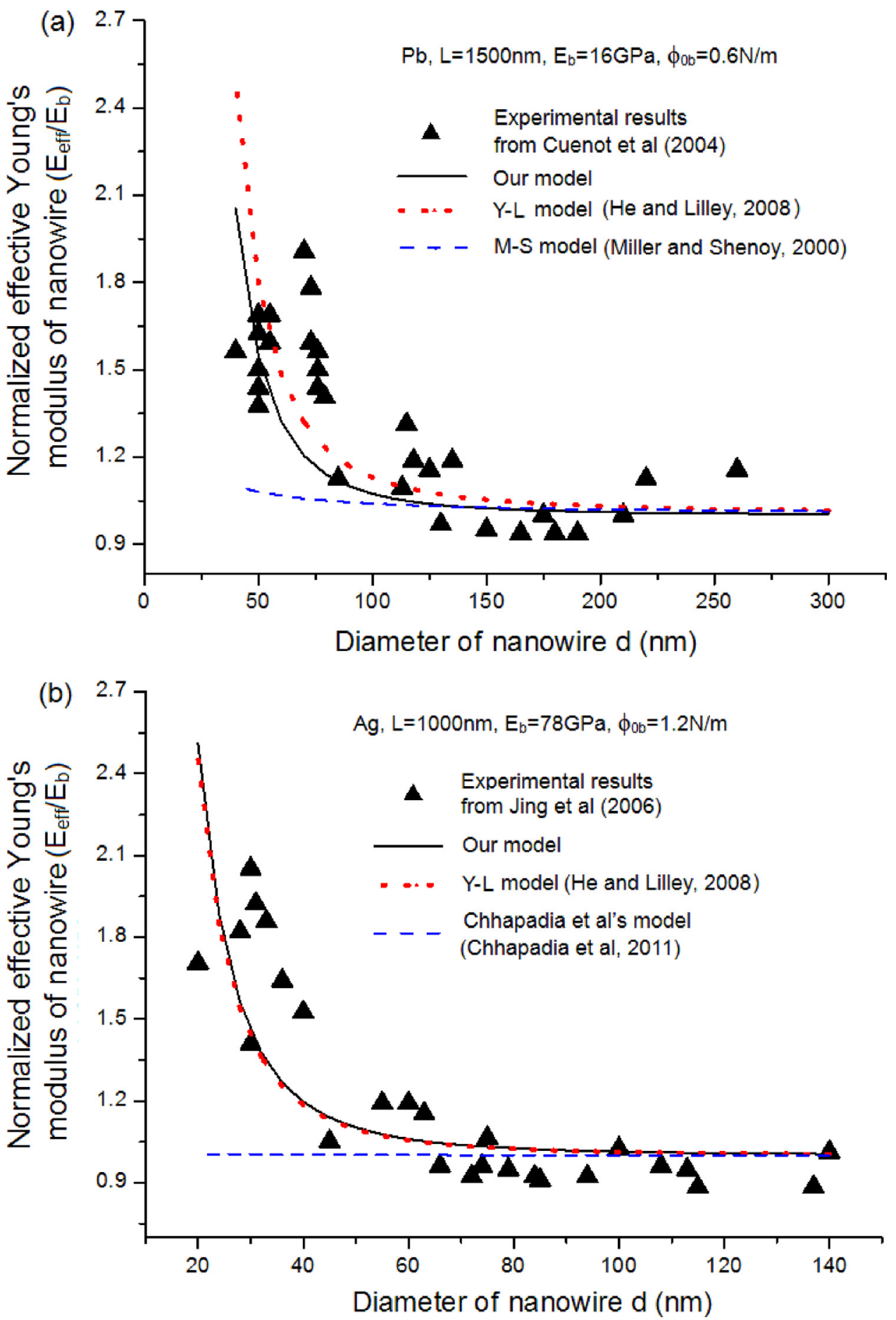

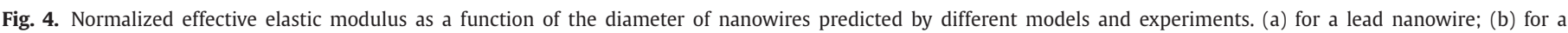
silver nanowire.

$E_{e f f}=E_{b}\left(1+\frac{E_{s} I_{s 1}}{E_{b} I}+\frac{D_{s} I_{c}}{E_{b} I}\right)$, (Chhapadia et al.'s model $)$

In the above formulae, $E_{S}$ denotes the surface elastic modulus, whose value was taken as $8 \mathrm{~N} / \mathrm{m}$ for $\mathrm{Pb}$ and $1.22 \mathrm{~N} / \mathrm{m}$ for $\mathrm{Ag}(\mathrm{He}$ and Lilley, 2008). $D_{S}$ is the surface bending modulus with a value of $0.344 \mathrm{~N} \cdot \mathrm{m}$ for Ag (Chhapadia et al., 2011). The effective elastic modulus predicted by Eqs. (36) and (37) is also shown in Figs. 4(a) and (b) for comparison, respectively. It shows that the above two equations can successfully predict the experimental results only when the diameter of nanowires is larger than $75 \mathrm{~nm}$ for $\mathrm{Pb}$ and $50 \mathrm{~nm}$ for $\mathrm{Ag}$.

\subsection{The case of a cantilever nanowire}

The bending experiment of a cantilever silicon nanowire was carried out by Sadeghian et al. (2010), in which the nanowire has a rectangular cross-section with a width $b=8 \mu \mathrm{m}$, an axial length $L=10 \mu \mathrm{m}$ and a height $h$ ranging from $40 \mathrm{~nm}$ to $1 \mu \mathrm{m}$. The theoretical prediction and the experimental measurement of the effective elastic modulus for cantilever nanowires are compared in 


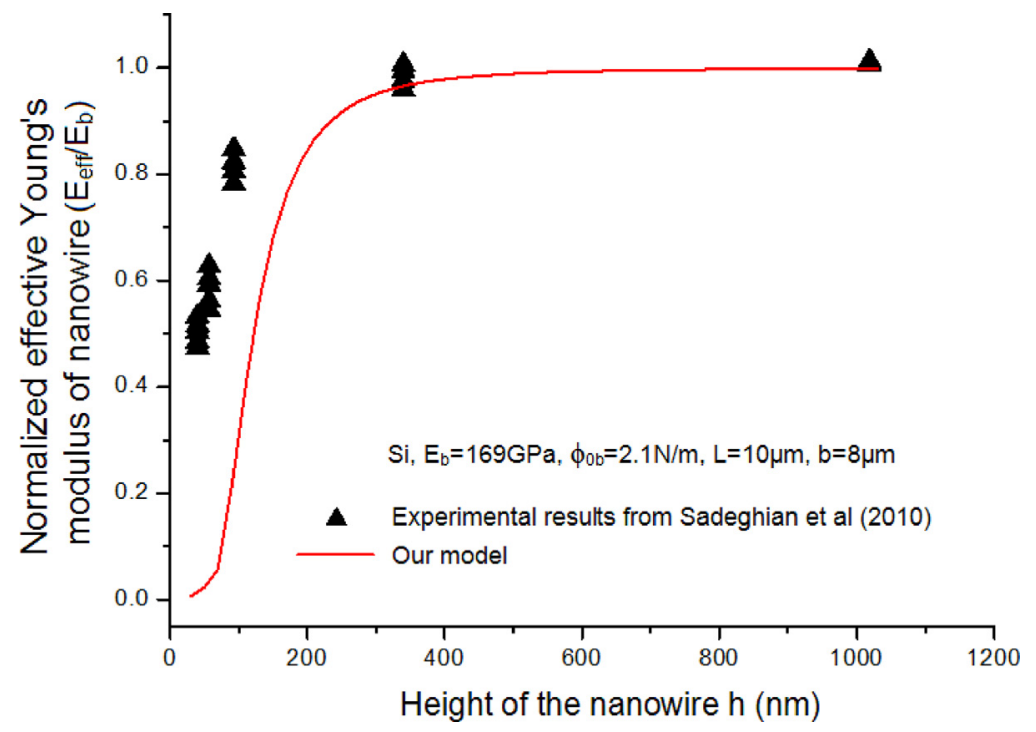

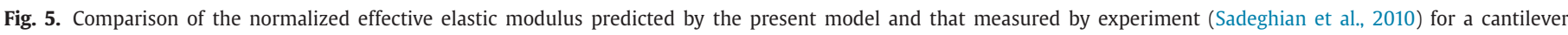
silicon nanowire.

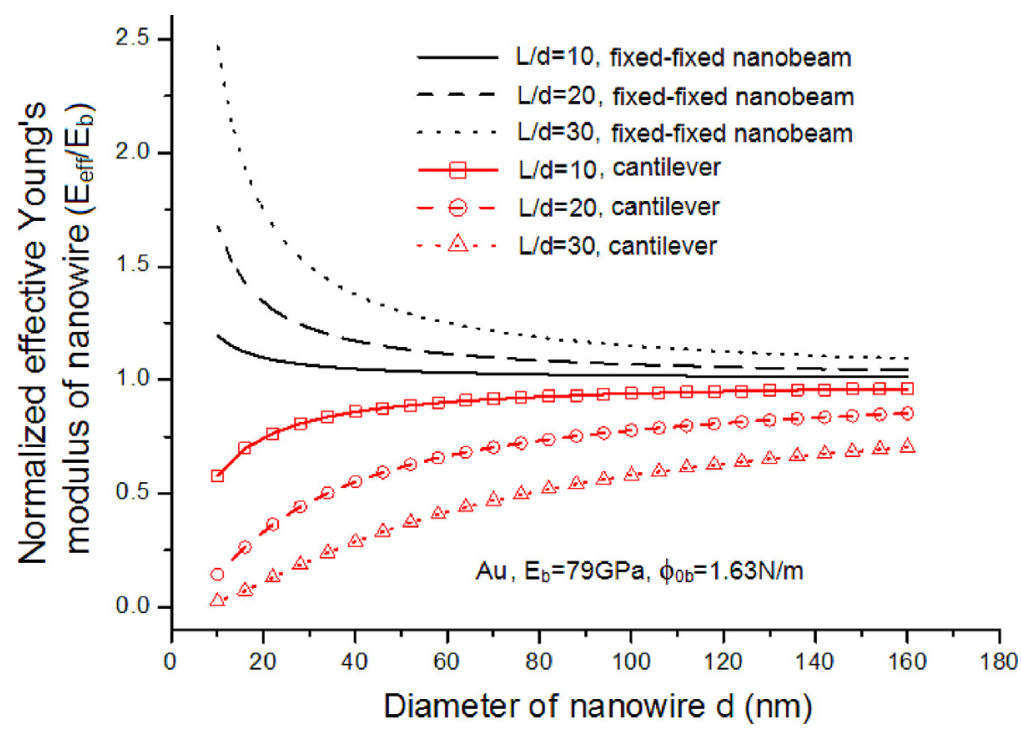

Fig. 6. Effective elastic modulus predicted theoretically as a function of the diameter of fixed-fixed nanowires and cantilever ones with different aspect ratios.

Fig. 5, where it shows that the effective elastic modulus $E_{\text {eff }}$ increases with an increasing height of nanowires and finally approaches the bulk value. It suggests that the reduction of a cantilever nanobeam height would lead to a softening behavior, contrary to the stiffening characteristic of a fixed-fixed nanowire. Such a softening behavior has also been observed by many experiments and numerical simulations (Park et al., 2005; Park and Klein, 2008; Gavan et al., 2009; Sadeghian et al., 2010; Mohammadi and Sharma, 2012).

Though the varying trend of the effective elastic modulus predicted by the present model is consistent well with that measured by experiments, an obvious difference between the theoretical result and the experimental one can be found in Fig. 5, especially for cantilever nanobeams with a relatively small height $(h \leq$ $100 \mathrm{~nm}$ ). A few aspects may be responsible for such a deviation. In the present theoretical model, $m=1$ is adopted for silicon materials though such a value is more appropriate for pure metals. The beam bending theory is adopted to analyze samples in the experiment (Sadeghian et al., 2010), though the sample looks more like a plate than a beam. Moreover, as mentioned by Sadeghian et al.,
(2010), the fabrication-induced defects and the native oxide layer within nanowire samples may also have important influences on the experimental measurements of stiffness, which, however, are not considered in the present model. All these issues will be further studied in our future work.

\subsection{Comparison of the two kinds of nanowires}

Comparing to the solution obtained by the classical beam theory, both the above theoretical prediction and the experimental measurement show that a fixed-fixed nanowire is always stiffened while a cantilever nanobeam is always softened. Fig. 6 gives the effective elastic modulus varying with the aspect ratio of both kinds of nanowires. The effective elastic modulus increases for a fixedfixed nanobeam, while decreases for a cantilever one with an increasing aspect ratio. It suggests that not only the nanoscale diameter but also the length would show significant influences on the elastic behavior of both kinds of nanowires, though this aspect was not noted by the existing experiment. The larger the aspect ratio, 
the stronger the stiffening of a fixed-fixed-nanobeam is, and the more obvious the softening of a cantilever nanowire is.

An interesting question is easily raised that why the surface effect in both kinds of nanowires is totally different. The physical discrepancy originates perhaps from the curvature. For a cantilever nanowire, an upward curvature results in a surface-induced traction with the same direction as that of the external load, leading to a larger deflection than the classical one. While a downward curvature in most parts of a fixed-fixed bending nanowire may give a surface-induced traction with an opposite direction to that of the external load, which acts as a resistance of the external load, yielding a smaller deflection than the classical one. As a result, a fixedfixed nanowire is stiffened while a cantilever nanowire is softened as compared to a bulk wire of the same material. However, the surface-induced traction in the normal direction of the surface can only affect the magnitude of the deflection, without bringing any additional rotation of the nanowire's cross-section. The assumption of an Euler-Bernoulli beam is still valid.

Nanowires in the present paper are regarded as Euler-Bernoulli beams without considering the shear deformation, which should be reasonable for a slender nanowire $(L / d \geq 10)$. For nanowires with a relatively small aspect ratio $(L / d<10)$, the Timoshenko beam model may be more accurate to describe the bending behavior (Timoshenko and Gere, 1972).

\section{Conclusion}

Based on a recently developed continuum theory for materials in nanoscale (Chen and Yao, 2014), the bending behavior of two kinds of nanowires with different boundary conditions is investigated. Closed-form solutions of the deflection and effective elastic modulus for both nanowires are obtained. It shows that the theoretical prediction agrees very well with the existing experimental measurement not only for a fixed-fixed nanowire but also for a cantilever one. Not only the characteristic length but also the aspect ratio exhibits significant effects on the elastic behavior of both nanowires. In contrast to the prediction of the classical beam theory, the surface effect will stiffen the elastic property of fixed-fixed nanowires, but soften that of cantilever ones. The smaller the characteristic length or the larger the aspect ratio, the larger the effective elastic modulus of a fixed-fixed nanobeam is, but the smaller the effective elastic modulus of a cantilever nanobeam will be. The physical origin may be due to the curvature, which leads to a surface-induced traction with the same or an opposite direction as or to that of the external load. This study may provide an efficient and convenient approach to study the surface effect in nanobeams and the result should be helpful for the design of nano-devices and nanomaterials.

\section{Acknowledgments}

The work reported here is supported by NSFC through grants \#11372317, \#11402270, \#11532013, the Nano-project (2012CB937500), and the CAS/SAFEA International Partnership Program for Creative Research Teams.

\section{Appendix}

Fig. A.1 shows a local coordinate system with axes 1 and 2 parallel to the two basic vectors of the surface unit cell and a global one $\{x, y, z\}$ attached to the nanowire. It is obvious that the local coordinate in (001) crystal surface does not coincide with the global one. The relation of the surface strain in bond directions and the strain induced by the external load in the global coordinate system can be established through a coordinate transformation,

$\varepsilon_{i j}^{\prime}=q_{k i} q_{l j} \varepsilon_{k l}$

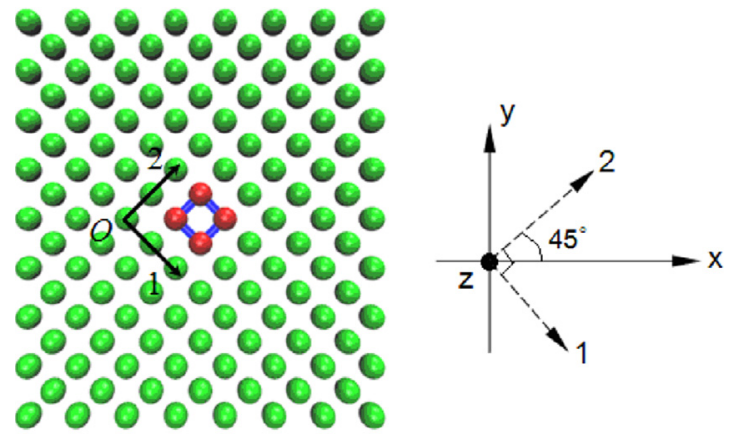

(001) surface

Fig. A.1. Schematic of a (001) surface and the relation between a surface local coordinate system $(1,2)$ and a global one $(x, y, z)$. The insetted quadrilateral consisting of four red atoms represents a surface unit cell.

where $q_{k i}$ is a component of the transformation tensor and the subscripts $i, j, k, l$ range from 1 to 2 .

For the bending of a nanobeam, we have $\varepsilon_{11}=\varepsilon_{x}, \varepsilon_{22}=\varepsilon_{12}=$ $\varepsilon_{21}=0$, where $\varepsilon_{i j}$ is the strain component in the global coordinate system and $\varepsilon_{x}$ is the axial strain of the nanobeam. $\varepsilon_{i j}^{\prime}$ represents the strain in the local coordinate system with $\varepsilon_{11}^{\prime}=\varepsilon_{s 1}, \varepsilon_{22}^{\prime}=\varepsilon_{s 2}$, where $\varepsilon_{11}^{\prime}$ and $\varepsilon_{22}^{\prime}$ are in the axes 1 and 2 directions, respectively.

On the (001) surface, an angle of $45^{\circ}$ exists between the local and global coordinate systems as shown in Fig. A.1. The components of the transformation tensor $q_{k i}$ are

$q_{11}=\sin 45^{\circ}, q_{12}=\cos 45^{\circ}, q_{21}=-\cos 45^{\circ}, q_{22}=\sin 45^{\circ}$ have

Expanding the coordinate transformation given by Eq. (A.1), we

$$
\begin{aligned}
\varepsilon_{s 1} & =q_{11}^{2} \varepsilon_{11}+q_{21}^{2} \varepsilon_{22}+2 q_{11} q_{21} \varepsilon_{12} \\
\varepsilon_{s 2} & =q_{12}^{2} \varepsilon_{11}+q_{22}^{2} \varepsilon_{22}+2 q_{12} q_{22} \varepsilon_{12} \\
\varepsilon_{s 12} & =q_{11} q_{12} \varepsilon_{11}+q_{21} q_{22} \varepsilon_{22}+\left(q_{11} q_{22}+q_{12} q_{21}\right) \varepsilon_{12}
\end{aligned}
$$

Substituting Eq. (A.2) into Eqs. (A.3) yields

$\varepsilon_{s 1}=\varepsilon_{s 2}=\varepsilon_{s 12}=\frac{\varepsilon_{x}}{2}$

\section{References}

Celik, E., Guven, I., Madenci, E., 2011. Mechanical characterization of nickel nanowires by using a customized atomic force microscope. Nanotechnology 22 (15), 155702.

Chan, W.K., Li, J.R., Wang, Y., Zhang, S.Y., Zhang, T.Y., 2010. Analysis of nanobridge tests. Acta Mech. Solida Sin. 23 (4), 283-296.

Chen, S.H., Wang, T.C., 2000. A new hardening law for strain gradient plasticity. Acta Mater 48 (16), 3997-4005.

Chen, S.H., Wang, T.C., 2002. A new deformation theory for strain gradient effects. Int. J. Plast. 18 (8), 971-995

Chen, S.H., Yao, Y., 2014. Elastic theory of nanomaterials based on surface energy density. ASME J. Appl. Mech. 81 (12), 121002.

Chen, T.Y., Chiu, M.S., 2011. Effects of higher-order interface stresses on the elastic states of two-dimensional composites. Mech. Mater. 43 (4), 212-221.

Chen, Y.X., Dorgan, B.L., Mcllroy, D.N., Aston, D.E., 2006. On the importance of boundary conditions on nanomechanical bending behavior and elastic modulus determination of silver nanowires. J. Appl. Phys. 100 (10), 104301.

Chhapadia, P., Mohammadi, P., Sharma, P., 2011. Curvature-dependent surface energy and implications for nanostructures. J. Mech. Phys. Solids 59 (10), 2103-2115.

Chiu, M.S., Chen, T.Y., 2011. Effects of high-order surface stress on static bending behavior of nanowires. Physica E 44 (3), 714-718.

Craighead, H.G., 2000. Nanoelectromechanical Systems. Science 290 (5496) 1532-1535.

Cuenot, S., Fretigny, C., Champagne, S.D., Nysten, B., 2004. Surface tension effect on the mechanical properties of nanomaterials measured by atomic force microscopy. Phys. Rev. B 69 (16), 165410.

Diao, J.K., Gall, K., Dunn, M.L., 2004. Atomistic simulation of the structure and elastic properties of gold nanowires. J. Mech. Phys. Solids 52 (9), 1935-1962.

Fleck, N.A., Hutchinson, J.W., 1993. A phenomenological theory for strain gradient effects in plasticity. J. Mech. Phys. Solids 41 (12), 1825-1857. 
Fleck, N.A., Hutchinson, J.W., 1997. Strain gradient plasticity. In: Hutchinson, J.W., Wu, T.Y. (Eds.). In: Advances in Applied Mechanics, 33. Academic Press, New York, pp. 295-361.

Gao, H., Huang, Y., 2001. Taylor-based nonlocal theory of plasticity. Int. J. Solids Struct. 38 (15), 2615-2637.

Gao, H., Huang, Y., Nix, W.D., Hutchinson, J.W., 1999. Mechanism-based strain gradient plasticity-I. Theory. J. Mech. Phys. Solids 47 (6), 1239-1263.

Gavan, K.B., Westra, H.J.R., Van der drift, E.W.J.M., Venstra, W.J., Van der zant, H.S.J., 2009. Surface effects on elastic properties of silver nanowires: Contact atomicforce microscopy. Appl. Phys. Lett. 94 (23), 233108.

Georgakaki, D., Ziogos, O.G., Polatoglou, H.M., 2014. Vibrational and mechanical properties of Si/Ge nanowires as resonators: A molecular dynamics study. Phys. Status Solidi A 211 (2), 267-276.

Gong, C.K., Liang, J.J., Wei, H., Niu, X.F., Ma, S.W., Hahn, H.T., Pei, Q.B., 2013. A Healable, semitransparent silver nanowire-polymer composite conductor. Adv. Mater. 25 (30), 4186-4191.

Gurtin, M.E., Murdoch, A.I., 1975. A continuum theory of elastic material surfaces. Arch. Rat. Mech. Anal. 57 (4), 291-323.

Gurtin, M.E., Murdoch, A.I., 1978. Surface stress in solids. Int. J. Solids Struct. 14 (6), 431-440.

He, J., Lilley, C.M., 2008. Surface Effect on the elastic behavior of static bending nanowires. Nano Lett 8 (7), 1798-1802.

Huang, Z.P., Wang, J., 2006. A theory of hyperelasticity of multi-phase media with surface/interface energy effect. Acta Mech 182 (3-4), 195-210.

Jaccodine, R.J., 1963. Surface energy of germanium and silicon. J. Electrochem. Soc. 110 (6), 524-527.

Jiang, Q., Liang, L.H., Zhao, D.S., 2001. Lattice contraction and surface stress of fcc nanocrystals. J. Phys. Chem. B 105 (27), 6275-6277.

Jing, G.Y., Duan, H.L., Sun, X.M., Zhang, Z.S., Xu, J., Li, Y.D., Wang, J.X., Yu, D.P., 2006. Surface effects on elastic properties of silver nanowires: Contact atomic-force microscopy. Phys. Rev. B 73 (23), 235409.

Lee, D.J., Park, H.S., Ryu, H.J., Jeon, S.K., Hong, S.H., 2011. Microstructure and mechanical properties of SiC-nanowire-augmented tungsten composites. J. Alloys Compd. 509 (37), 9060-9064.

Li, X.F., Zhang, H., Lee, K.Y., 2014. Dependence of Young's modulus of nanowires on surface effect. Int. J. Mech. Sci. 81, 120-125.

Liang, H.Y., Upmanyu, M., 2005. Size-dependent elasticity of nanowires: Nonlinear effects. Phys. Rev. B 71 (24), 241403.

Liang, L.H., You, X.M., Ma, H.S., Wei, Y.G., 2010. Interface energy and its influence on interface fracture between metal and ceramic thin films in nanoscale. J. Appl. Phys. 108 (8), 084317.

Mi, C.W., Jun, S., Kouris, D.A., Kim, S.Y., 2008. Atomistic calculations of interface elastic properties in noncoherent metallic bilayers. Phys. Rev. B 77 (7), 075425.

Miller, R.E., Shenoy, V.B., 2000. Size-dependent elastic properties of nanosized structural elements. Nanotechnology 11 (3), 139-147.

Mohammadi, P., Sharma, P., 2012. Atomistic elucidation of the effect of surface roughness on curvature dependent surface energy, surface stress, and elasticity. Appl. Phys. Lett. 100 (13), 133110.

Nam, C.Y., Jaroenapibal, P., Tham, D., Luzzi, D.E., Evoy, S., Fischer, J.E., 2006. Diameter-dependent electromechanical properties of GaN nanowires. Nano. Lett 6 (2), 153-158.
Nix, W.D., Gao, H., 1998. An atomic interpretation of interface stress. Scr. Mater. 39 (12), 1653-1661.

Olsson, P.A.T., Park, H.S., 2012. On the importance of surface elastic contributions to the flexural rigidity of nanowires. J. Mech. Phys. Solids 60 (12), 2064-2083.

Ouyang, G., Tan, X., Yang, G.W., 2006. Thermodynamic model of the surface energy of nanocrystals. Phy. Rev. B 74 (19), 195408.

Ouyang, G., Li, X.L., Tan, X., Yang, G.W., 2008. Surface energy of nanowires. Nanotechnology 19 (4), 045709.

Park, S.H., Kim, J.S., Park, J.H., Lee, J.S., Choi, Y.K., Kwon, O.M., 2005. Molecular dynamics study on size-dependent elastic properties of silicon nanocantilevers. Thin Solid Films 492 (1-2), 285-289.

Park, H.S., Klein, P.A., 2008. Surface stress effects on the resonant properties of metal nanowires: The importance of finite deformation kinematics and the impact of the residual surface stress. J. Mech. Phys. Solids 56 (11), 3144-4166.

Sadeghian, H., Yang, C.K., Goosen, J.F.L., Van der Drift, E., Bossche, A., French, P.J., Van Keulen, F., 2009. Characterizing size-dependent effective elastic modulus of silicon nanocantilevers using electrostatic pull-in instability. Appl. Phys. Lett. 94 (22), 221903.

Sadeghian, H., Yang, C.K., Goosen, J.F.L., Bossche, A., Staufer, U., French, P.J., Van Keulen, F., 2010. Effects of size and defects on the elasticity of silicon nanocantilevers. Nanotechnology 20 (6), 064012.

Sheng, H.W., Kramer, M.J., Cadien, A., Fujita, T., Chen, M.W., 2011. Highly optimized embedded-atom-method potentials for fourteen fcc metals. Phys. Rev. B 83 (13), 134118.

Shenoy, V.B., 2005. Atomistic calculations of elastic properties of metallic fcc crystal surfaces. Phys. Rev. B 71 (9), 094104.

Song, F., Huang, G.L., Park, H.S., Liu, X.N., 2011. A continuum model for the mechanical behavior of nanowires including surface and surface-induced initial stresses. Int. J. Solids Struct. 48 (14-15), 2154-2163.

Steigmann, D.J., Ogden, R.W., 1997. Plane deformations of elastic solids with intrinsic boundary elasticity. Proc. R. Soc. Lond. A 453 (1959), 853-877.

Sun, C.Q., 2003. Oxidation electronics: bond-band-barrier correlation and its applications. Prog. Mater. Sci. 48 (6), 521-685.

Tan, E.P.S., Zhu, Y., Yu, T., Dai, L., Sow, C.H., Tan, V.B.C., Lim, C.T., 2007. Crystallinity and surface effects on Young's modulus of $\mathrm{CuO}$ nanowires. Appl. Phys. Lett. 90 (16), 163112.

Timoshenko, S.P., Gere, J.M., 1972. Mechanics of Materials. Van Nostrand Reinhold Co., New York

Wang, G.F., Feng, X.Q., 2007. Effects of surface elasticity and residual surface tension on the natural frequency of microbeams. Appl. Phys. Lett. 90 (23), 231904.

Wang, Z.J., Liu, C., Li, Z.G., Zhang, T.Y., 2010. Size-dependent elastic properties of Au nanowires under bending and tension-Surfaces versus core nonlinearity. J. Appl. Phys. 108, 083506.

Wolfer, W.G., 2011. Elastic properties of surfaces on nanoparticles. Acta Mater 59 (20), 7736-7743.

Xie, P., Xiong, Q.H., Fang, Y., Qing, Q., Lieber, C.M., 2012. Local electrical potential detection of DNA by nanowire-nanopore sensors. Nat. Nanotechnol. 7, 119-125.

Zhang, W.X., Wang, T.J., Chen, X., 2010. Effect of surface/interface stress on the plastic deformation of nanoporous materials and nanocomposites. Int. J. Plast. 26 (7), 957-975. 